NASA Contractor Report 4478

\title{
Design Optimization of Natural Laminar Flow Bodies in Compressible Flow
}

Simha S. Dodbele

CONTRACT NAS1-18585

DECEMBER 1992 



\section{Design Optimization of Natural Laminar Flow Bodies in Compressible Flow}

Simha S. Dodbele

ViGYAN, Inc.

Hampton, Virginia

Prepared for

Langley Research Center

under Contract NAS1-18585

\section{N/SA}

National Aeronautics and

Space Administration

Office of Management

Scientific and Technical

Information Program 


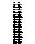




\title{
DESIGN OPTIMIZATION OF NATURAL LAMINAR FLOW BODIES IN COMPRESSIBLE FLOW
}

\author{
Simha S. Dodbele \\ ViGYAN, Inc., Hampton, VA
}

\begin{abstract}
An optimization method has been developed to design axisymmetric body shapes such as fuselages, nacelles and external fuel tanks with increased transition Reynolds numbers in subsonic compressible flow. The new design method involves a constraint minimization procedure coupled with analysis of the inviscid and viscous flow regions and linear stability analysis of the compressible boundary-layer. In order to reduce the computer time, Granville's transition criterion is used to predict boundarylayer transition and to calculate the gradients of the objective function, and linear stability theory coupled with the $e^{n}$-method is used to calculate the objective function at the end of each design iteration. Use of the method to design an axisymmetric body with extensive natural laminar flow is illustrated through the design of a tiptank of a business jet. For the original tiptank, boundary layer transition is predicted to occur at a transition Reynolds number of $6.04 \times 10^{6}$. For the designed body shape, a transition Reynolds number of $7.22 \times 10^{6}$ is predicted using compressible linear stability theory coupled with the $\mathrm{e}^{\mathrm{n}}$-method.
\end{abstract}

\section{INTRODUCTION}

Advances in airplane construction techniques and materials, such as bonded and milled aluminum skins and composite materials, enable production of aerodynamic surfaces without significant waviness and roughness, thus permitting long runs of natural laminar flow (NLF) over wings in subsonic flow. These advances provide excellent opportunities for airplane drag reduction by increasing the extent of NLF over wings [1]. Compared to lifting surfaces, laminar flow research on nonlifting airframe surfaces such as fuselages, nacelles, and external fuel tanks has received limited attention [2,3]. Substantial drag reduction, however, can be obtained by increasing the extent of laminar flow on the nonlifting 
component surfaces. A recent study [4] of bodies of revolution at high subsonic speeds without supersonic regions (subcritical flow) demonstrated the potential for tripling the length of sufficiently stable laminar flow at a Mach number, $\mathrm{M}$, of 0.8 and a length Reynolds number, $\mathrm{R}_{\mathrm{L}}$, of $40 \times 10^{6}$, in comparison with incompressible speed at the same length Reynolds number.

Reference [3] presents a recent overview of incompressible transition experiments on axisymmetric body shapes. References [5-7] presented results of mostly incompressible, underwater transition experiments on bodies of revolution with varying fineness ratios indicating maximum transition Reynolds numbers of about 20 million. A transition experiment was conducted in the NASA-Ames 12-ft. pressure tunnel by Boltz, et al., [8, 9] at high subsonic freestream Mach numbers, measuring the transition locations on two ellipsoids of fineness ratios of 7.5 and 9.14. Transition occurred near the location 80 to $88 \%$ of the length of the body aft of the nose at $\mathrm{M}=0.90$ to 0.96 . Reference 10 presents correlation of compressible boundary-layer-stability analysis for several of the experimental results reported by Boltz et al,, and indicates that integrated $T-S$ linear logarithmic amplification factors (n-factors) of 8-11 are obtained at the point of measured transition onset.

Transition over an axisymmetric body shape, where crossflow is minimal, is usually caused by the growth of Tollmien-Schlichting (T-S) disturbance waves in the laminar boundary layer. In compressible flow, the presence of density gradient in the boundary layer in the direction normal to the wall in addition to the velocity gradients can result in a large reduction in the spatial growth of $T-S$ disturbances in the laminar boundary layer. The favorable damping effect of the $T-S$ waves in compressible flow contributes to the achievement of increased transitionReynolds numbers on lifting as well as nonlifting aircraft surfaces in the absence of strong crossflow [4]. This favorable effect of compressibility can be exploited in the design of advanced NLF bodies for application to general aviation, commuter, transport aircraft and business jets.

This paper presents a design method to generate body shapes with increased transition Reynolds numbers at subsonic compressible speeds. In refs. [2 and 3] it was indicated that a transition-prediction criterion based on linear boundary-layer stability theory coupled with the $\mathrm{e}^{\mathrm{n}}$ method (originally introduced by Smith [11] and Van Ingen [12]) appeared to be a 
more reliable approach to predict the onset of transition in the design of NLF fuselages [13-16]. However, the prediction of onset of transition using only linear stability analysis coupled with the $e^{n}$-method (henceforth referred to as the $e^{n}$-method) in the design method can be very expensive and time consuming. A new approach has been formulated in the design method in which Granville's transition criterion [17] has been used for predicting the gradients of the objective function in the design calculations and the $e^{n}$-method has been used only while predicting the objective function at the end of each iteration. This new approach may be more practical in the design calculations. This report presents design calculations for a tiptank in compressible flow as an example case.

\section{NOMENCLATURE}

$\mathrm{A} / \mathrm{A}_{0} \quad$ Ratio of local disturbance amplitude to amplitude at the point of neutral stability for a fixed disturbance frequency

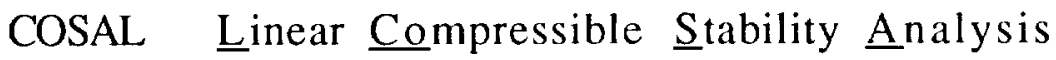

$\mathrm{C}_{\mathrm{D}} \quad$ Body drag coefficient (based on frontal area)

$\mathrm{C}_{\mathrm{p}} \quad$ Pressure coefficient

$f_{\mathrm{r}} \quad$ Fineness ratio (body length/maximum body diameter)

$f_{\text {obj }} \quad$ Objective function

L Body length, $\mathrm{ft}$

M Freestream Mach number

NLF $\quad$ Natural Laminar $\underline{\text { Flow }}$

n Logarithmic exponent of amplitude-growth ratio of unstable Tollmien-Schlichting wave, $\mathrm{n}=\ln \left(\mathrm{A} / \mathrm{A}_{0}\right)$

$\mathrm{R}_{\mathrm{L}} \quad$ Reynolds number based on freestream conditions and body length 
$\mathrm{R}_{\mathrm{tr}} \quad$ Reynolds number based on freestream conditions and transition length

R' Unit Reynolds number based on freestream conditions

T-S Tollmien-Schlichting

$\mathrm{T}(\mathrm{y}) \quad$ Boundary-layer temperature profile, ${ }^{0} \mathrm{~F}$

$\mathrm{U}(\mathrm{y}) \quad$ Boundary-layer velocity profile, $\mathrm{ft} / \mathrm{sec}$

$\mathrm{V} \quad$ Volume of the body, cu. $\mathrm{ft}$

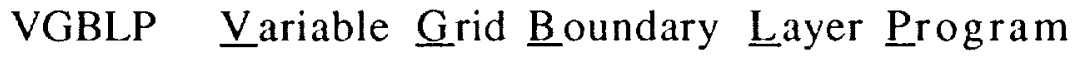

VSAERO Subsonic AEROdynamic Characteristics of Arbitrary Configuration Having Vortex Separation and Strong Vortex/ Surface Interaction

$\mathrm{X} \quad$ Axial coordinate starting at nose, $\mathrm{ft}$

$\mathrm{X}_{\mathrm{tr}} \quad$ Axial coordinate of transition starting at nose, $\mathrm{ft}$

$\mathrm{x} \quad$ Nondimensional axial coordinate, $\mathrm{X} / \mathrm{L}$

$x_{t r} \quad$ Nondimensional axial coordinate of transition, $x_{t r} / L$

$\mathrm{x}_{\mathrm{tr}}(\mathrm{g}) \quad$ Nondimensional transition location by Granville's criterion

$x_{t r}\left(e^{n}\right)$ Nondimensional transition location by $e^{n}$-method

$\alpha \quad$ Angle of attack, deg

$\psi \quad$ Obliqueness of $\mathrm{T}-\mathrm{S}$ disturbances with respect to streamlines, deg

2-D Two-dimensional flow 


\section{OPTIMIZATION PROCEDURE FOR NLF BODY DESIGN}

A flow chart of the design method developed to obtain axisymmetric body shapes with extensive runs of laminar flow is presented in Fig. 1. ${ }^{1}$ Initial values of the design variables in the form of body ordinates describing the body shape are input along with the length Reynolds number, Mach number of the free stream, and the fineness ratio of the desired body shape (The superscript at the beginning of a word refers to the block number in Fig. 1). The axisymmetric body is described by design variables representing the body ordinates in the forebody section and in the aftbody section. By having a large number of design variables in the forebody region, the forebody can be represented in great detail especially near the nose region [18] and such detailed representation can incorporate small body perturbations dictated by the design cycles.

There are several methods available for optimization in engineering applications, e.g., the constrained minimization method [19], quasiNewton's method [20], and evolution method [21]. ${ }^{2}$ The constrained minimization method developed by Vanderplaats (CONMIN) [19] was used in the design procedure because of its easy accessibility. The constrained minimization method is coupled with analysis of the ${ }^{3}$ inviscid and 4 viscous flow regions, ${ }^{5}$ linear stability analysis of the compressible boundary-layer, and a transition prediction method.

The ${ }^{3}$ aerodynamic analysis program used in the optimization procedure is based on a low-order surface-singularity method (VSAERO, [22]). Pressure distributions and velocity distributions are computed by this aerodynamics program which uses surface singularity panels to represent the body shape. The ${ }^{4}$ boundary-layer profiles along the surface of the body, required for the $\mathrm{e}^{\mathrm{n}}$-method, are generated by a modified axisymmetric boundary layer code (VGBLP, [23]). The finite difference program VGBLP calculates detailed boundary-layer velocity and temperature profiles along with their first and second derivatives normal to the surface, including the effects of transverse curvature. Compressible linear stability theory is used to analyze the laminar boundary-layer stability along the body via the COSAL program [24]. The COSAL program solves the finite-differenced boundary-layer stability equations by matrix methods. The compressible T-S eigenvalue problem is solved for each boundary-layer station along the body surface, giving temporal growth 
rates of the instability waves propagating at specific wavelengths and wave angles. The temporal growth rates are transformed to spatial growth rates using Gaster's phase-velocity relationship [25]. Boundary-layer transition is predicted by the $\mathrm{e}^{\mathrm{n}}$-method in which $\mathrm{n}$, usually referred to as $\mathrm{n}$-factor, is obtained by integrating the linear growth rate of the $\mathrm{T}$-S waves from the neutral stability point to a location downstream of the body.

The correlation of a large number of wind-tunnel data and flight transition experiments with linear boundary-layer stability calculations has made the $\mathrm{e}^{\mathrm{n}}$-method an accepted transition-prediction method (see [15]). For experiments in wind tunnels with low turbulence and low acoustic levels, the onset of transition can be correlated with an $\mathrm{n}$-factor of 9 to 11 in subsonic, transonic and supersonic flows. In the case of flight tests conducted at low Mach numbers, higher n-factors on the order of 12 to 15 have been observed to correlate with transition; for compressible flight conditions $\mathrm{n}$-factors of 9 or lower have generally correlated with transition.

A number of geometric and aerodynamic constraints are imposed on the design parameters to generate practical and realistic body shapes for given design conditions. Judicious choice of the upper and the lower bounds for the design variables will accelerate convergence of the solutions. Both the level and the location of the minimum surface pressure along the body surface are aerodynamically constrained by the requirement that the turbulent boundary layer over the aft-portion of the body should not separate until $x=0.95$ for the design conditions.

Next, an 7 objective function is chosen and minimized subject to the geometric and aerodynamic constraints. The objective function $f_{o b j}$ is taken to be a function of the location of transition as follows

$$
\begin{aligned}
& \mathrm{f}_{\mathrm{obj}}=1-\mathrm{x}_{\mathrm{tr}}(\mathrm{g}) \quad \begin{array}{l}
\text { for calculating gradients of } \\
\text { the objective function, }
\end{array} \\
& \mathrm{f}_{\mathrm{obj}}=1-\mathrm{x}_{\mathrm{tr}}\left(\mathrm{e}^{\mathrm{n}}\right) \quad \begin{array}{l}
\text { for calculating the objective function } \\
\text { from a proposed set of design variables } \\
\text { at the end of each iteration, }
\end{array}
\end{aligned}
$$




$$
\begin{aligned}
& \text { where } x_{t r}(g) \quad \begin{array}{l}
\text { is the transition location predicted } \\
\text { using Granville's transition criterion }
\end{array} \\
& \text { and } x_{t r}\left(e^{n}\right) \quad \begin{array}{l}
\text { is the transition location predicted } \\
\text { using the } e^{n} \text {-method with an } n \text {-factor of } 9 .
\end{array}
\end{aligned}
$$

In the optimization calculations described in reference [2], the drag coefficient was chosen as the objective function. In the present design method the location of transition appears explicitly in the objective function, thus it is possible to derive a body optimized for NLF directly. The objective function given by Eqn. (1) is to be minimized subject to the constraints on the design variables. The optimizer computes ${ }^{6}$ gradients of the objective function using Granville's transition criterion; and then, using either a conjugate direction method or a method of feasible direction, the optimizer determines a linear search direction along which a new constrained variable is constructed.

An improved or minimum feasible value of the objective function is calculated using the $\mathrm{e}^{\mathrm{n}}$-method transition criterion given by Eqn. (1) and a series of revised design variables are calculated. The objective function and the constrained function are evaluated using the revised design variables, interpolating over the specified range of feasible design variables resulting in a minimum value of the objective function.

8 The results are tested against a convergence criterion. The procedure will stop if the convergence criterion is satisfied, giving a body shape with maximum transition length satisfying the separation constraint. If the convergence criterion is not satisfied the design parameters go through the optimizer again resulting in a new set of design variables and the procedure is repeated until a final body shape is obtained.

\section{COMPUTATIONAL RESULTS AND DISCUSSIONS}

Results obtained by the optimization procedure are discussed through an example. All the computations were performed using a CRAY$2 S$ computer. A body of revolution whose maximum diameter and length correspond to those of a tiptank of a representative business aircraft is considered. The tiptank has a fineness ratio of 8.00 and the design flight 
conditions considered for the present calculations are given by $\mathbf{M}=0.7$, zero incidence and $R^{\prime}=1.28 \times 10^{6}$ /foot. In the present example an $n$-factor of 9 is assumed for prediction of the onset of transition.

At zero incidence, provided that premature flow separation has not occured, the transition process is dominated by the growth of the 2-D, T-S disturbances prior to the inflectional instability that develops at minimum pressure. Crossflow vortex structure can develop on the body at nonzero angles of attack. These crossflow vortices can interact with $T$-S waves and may lead to premature transition. Such complex three-dimensional boundary-layer flows were not considered here.

The axisymmetric body was modelled by a set of 27 body coordinates with 12 points defining the forebody section and 15 points defining the aftbody section. For the aerodynamic analysis, the body was modelled by 32 panels in the axial direction and 8 panels in the circumferential direction resulting in a total of 256 panels. This number of panels produced reasonably accurate inviscid pressure distributions by the VSAERO method. The boundary-layer velocity and temperature profiles were obtained at 101 points in the direction normal to the surface and at 90 stations in the streamwise direction. Boundary layer calculations were carried out for adiabatic wall conditions and zero suction through the wall.

The boundary-layer stability equations were solved at every $5^{\text {th }}$ streamwise boundary-layer station starting from the first station. The boundary-layer stations were skipped to reduce the computation time. In the global search for eigenvalues, the sixth-order stability equation, obtained by neglecting dissipation terms in the boundary-layer stability equations, was solved at each chordwise station; and in the local search for the eigenvalues, the full eighth order stability equation was solved.

Figure 2 presents the pressure distribution on the baseline tiptank and the results of the compressible linear stability analysis for several T-S disturbance frequencies. The pressure coefficients remain subcritical on the entire body. The figure shows that at transition an $n$-factor of 9 occurs at a T-S disturbance frequency of $3500 \mathrm{~Hz}$. For this tiptank shape transition onset was predicted to occur at $x_{t r}\left(e^{n}\right)=0.327\left(R_{t r}\left(e^{n}\right)=6.04 x\right.$ $\left.10^{6}\right)$. 
For the tiptank shape twelve design variables representing the ordinates in the forebody region were allowed to vary within the set of specified upper and lower bounds while simultaneously holding the tail section aft of the maximum thickness point unchanged during the design iterations. In pure axisymmetric flow the obliqueness of the T-S waves with respect to the streamlines $(\psi)$ does not occur until the local flow speed exceeds the local sonic speed. In the present example, since the axisymmteric flow was subcritical, $\psi=0 \mathrm{deg}$ was assumed in the design calculations. Prior knowledge of the critical boundary-layer disturbance frequencies, which are functions of the Mach number, helps to identify the critical frequency spectrum during the course of the design optimization. The range of T-S disturbance frequencies should be chosen such that it does not exclude any disturbance frequencies which may grow substantially on a new body shape generated during the design iterations. In the present example, the body shapes did not go through large perturbations as the number of iterations increase, and hence, the initially chosen set of $T-S$ frequencies contained all the growing disturbances throughout the design calculations. The same set of T-S disturbance frequencies which are used in the linear stability analysis for the original tiptank was used in the design optimization.

The COSAL code [24] does not include transverse-curvature effects and the effect of streamline divergence (i.e., T-S wave stretching in the nose region-generally termed as vortex stretching, [26]). In general, transverse surface curvature has a slightly stabilizing influence on T-S disturbance growth if the curvature is large in relation to the thickness of the boundary layer [27]. Vortex stretching effects are considerably less if the body has a very small nose radius. If the design constraints are such that a body with a large nose radius is desired, the vortex stretching effects have to be included in the boundary layer stability analysis. The present design calculations do not take into account the effect of the vortex stretching. During the course of the design calculations, concave curvature may develop on the axisymmetric body. It is known that centrifugal instabilities in the form of Gortler vortices occur in the shear flow over concave surfaces $[28,29]$. In the present design method the boundary layer stability calculations do not consider the effect of the centrifugal instabilities. The design program took 2785 seconds on CRAY$2 S$ to predict the final tiptank design shape.

The original and the final tiptank shapes obtained are compared in Fig. 3. The stability envelope for the new tank has a smaller gradient than 
the original tiptank. On the original tiptank, high frequency disturbances $(5000 \mathrm{~Hz}, 6000 \mathrm{~Hz}$ and $7500 \mathrm{~Hz}$ ) grow along the first $20 \%$ of the body and then become stable. These high frequency disturbances do not grow beyond an $\mathrm{n}$-factor of 5 . The critical disturbances, characterized by a frequency of $3500 \mathrm{~Hz}$, start growing near $13 \%$ of the body length from the nose and reach an $n$-factor of 9 at $x_{t r}\left(e^{n}\right)=0.327\left(R_{t r}\left(e^{n}\right)=6.04 \times 10^{6}\right)$.

On the designed body, the disturbances with frequencies of $3500 \mathrm{~Hz}$ and $4000 \mathrm{~Hz}$ do not grow as much as they did on the original tiptank. The disturbance at a frequency of $9000 \mathrm{~Hz}$, which did not grow at all on the original tiptank, grows on the new body until an $n$-factor of about 6 is reached. The disturbance with a frequency of $2500 \mathrm{~Hz}$ does not grow at all on the designed body shape; whereas, the disturbance with the same frequency grew to an $\mathrm{n}$-factor of about 13 for the original tiptank. The transition location corresponding to an $n$-factor of 9 occurs at $x_{t r}\left(e^{n}\right)=$ $0.391\left(\mathrm{R}_{\mathrm{tr}}=7.22 \times 10^{6}\right)$ on the designed body. Although the critical frequency leading to transition remained at $3500 \mathrm{~Hz}$ on both the original tiptank and the designed body, boundary-layer transition corresponding to an $n$-factor of 9 as predicted by the $e^{n}$-method occured much further downstream on the designed body.

To assess the effect of extending the length of laminar boundarylayer flow over the geometries analyzed, The viscous drag was calculated using a modified integral boundary layer approach [3]. An improved turbulent boundary-layer calculation method was incorporated in the panel method to give more realistic drag calculations with available experimental data. The turbulent boundary-layer calculations were based on Head's entrainment method as modified by Shanebrook and Sumner [30]. Validation of this improved method with experimental drag measurements by Gërtler [31] on axisymmetric turbulent body shapes at $\mathrm{R}_{\mathrm{L}}$ of $10.0 \times 10^{6}$ and $26.0 \times 10^{6}$ show that the drag coefficient can be calculated accurately for these predominantly turbulent flow geometries [3]. A drag coefficient $\left(C_{D}\right)$ of 0.0491 was predicted on the original tiptank with the boundary-layer transition fixed at $x_{t r}=0.327$, and a drag coefficient $\left(C_{D}\right)$ of 0.0415 was predicted on the designed body shape with the boundary-layer transition fixed at $\mathrm{x}_{\mathrm{tr}}=0.391$. 
The designed body shape has a very small nose radius. Nose shapes with extremely small nose radii will produce large pressure peaks at offdesign angles of attack and can produce vortices which would influence transition in a negative way. A constraint on the nose radius will help alleviate the off-design aerodynamic characteristics.

\section{CONCLUDING REMARKS}

An optimization procedure has been developed to design axisymmetric body shapes with increased transition Reynolds numbers. The design method involves a constraint minimization procedure coupled with analysis of the inviscid and viscous flow regions and linear stability analysis of the compressible boundary-layer. Granville's criterion was used to compute all the gradients required by the constrained minimization method. At the end of each iteration, the objective function was predicted by a transition criterion based on the $e^{n}$-method. This new approach to evaluate the objective function would be more practical and would save computation time compared to the approach in which the $e^{n}$ method is used for all the transition predictions in the design method.

A tiptank of a business-jet was used to demonstrate the use of the method to design an axisymmetric body shape with increased transition Reynolds number. Boundary-layer transition was predicted to occur at a transition Reynolds number of $6.04 \times 10^{6}$ on the original tiptank and at $7.22 \times 10^{6}$ on the designed body shape. This was an increase of $20 \%$ in transition Reynolds number.

\section{Acknowledgment}

The research was supported by NASA Langley Research Center under NASA Contract NAS1-18585 to ViGYAN, Inc., Hampton, Virginia.

\section{REFERENCES}

[1] Holmes, B.J.; Obara, C.J.; and, Yip, L.P.: "Natural Laminar Flow Experiments on Modern Airplane Surfaces." NASA TP-2256, June 1984. 
[2]. Dodbele, S.S.; van Dam, C.P.; and Vijgen, P.M.H.W.: "Design of Fuselage Shapes for Natural Laminar Flow." NASA CR-3970, March 1986.

[3] Dodbele, S.S.; van Dam, C.P.; Vijgen, P.M.H.W.; and Holmes, B.J.: "Shaping of Airplane Fuselages for Minimum Drag." Journal of Aircraft, Vol. 24, May 1987, pp. 298-304.

[4] Vijgen, P.M.H.W.; Dodbele, S.S.; Holmes, B.J.; and van Dam, C.P.: "Effects of Compressibility on Design of Subsonic Fuselages for Natural Laminar Flow.," Journal of Aircraft, Vol. 25, Sept. 1988, pp. 776-782.

[5] Carmichael, B.H.: "Underwater Vehicle Drag Reduction through Choice of Shape." AIAA paper No. 66-657, 1966.

[6] Carmichael, B.H. and Niehuss,O.: "Computer Study To Establish the Lower Limit of Length-to-Diameter Rates Advisable for Low-Drag Bodies." SID 64-1938, North American Aviation, Inc., October 1964. (Available from DTIC as AD 664 610.)

[7] Carmichael, B.H.: "Underwater Drag Reduction Through Optimal Shape." In Underwater Missile Propulsion, edited by L. Greiner, Compass Publications, Inc., Arlington, VA, 1966.

[8] Boltz, F.W.; Kenyon, G.C.; and Allen, C.Q.: "The Boundary-Layer Transition Characteristics of Two Bodies of Revolution, A Flat Plate, and an Unswept Wing in a Low-Turbulence Wind Tunnel." NASA TN D-309, April 1960.

[9] Boltz, F.W.; Kenyon, G.C.; and Allen, C.Q.: "Measurements of BoundaryLayer Transition at Low Speeds on Two Bodies of Revolution in a Low-Turbulence Wind Tunnel." NACA RM A56G17, Sept. 1956.

[10] Vijgen, P.M.H.W.; Dodbele, S.S.; Pfenninger, W.; and Holmes, B.J.: "Analysis of Wind-Tunnel Boundary-Layer Transition Experiments on Axisymmetric Bodies at Transonic Speeds Using Compressible Boundary Layer Stability Theory." AIAA Paper No. 88-0008, 1988.

[11] Smith, A.M.O.: "Transition, Pressure Gradient and Stability Theory." IX International Congress for Applied Mechanics, Brussels, 1956. 
[12] Van Ingen, J.L.: " A Suggested Semi-Empirical Method for the Calculation of the Boundary Layer Transition Region." University of Technology, Department of Aerospace Engineering, VTH-74, Delft, 1956.

[13] Hefner, J.N.; and Bushnell, D.M.: "Status of Linear Boundary-Layer Stability Theory and the $\mathrm{e}^{\mathrm{n}}$-Method, With Emphasis on Swept-Wing Applications." NASA TP-1645, Feb. 1980.

[14] Jaffe, N.A.; Okamura, T.T.; and Smith, A.M.O.: "Determination of Spatial Amplification Factors and their Application to Predicting Transition." AIAA Journal, Vol. 8, No. 2, 1970, pp. 301-308.

[15] Bushnell, D.M.; Malik, M.R.; and Harvey, W.D.: "Transition Prediction in External Flows via Linear Stability Theory." IUTAM Symposium Transonicum III, Gottingen, West Germany, May 24-27, 1988.

[16] Malik, M.R.: "Instability and Transition in Supersonic Boundary Layers." Laminar Turbulent Boundary Layers (Eds: E.M.Uram and H.E. Weber), Energy Resources Technology Conference, New Orleans, Louisiana, Feb 12-16, 1984.

[17] Granville,P.S.: "The Calculation of the Viscous Drag of Bodies of Revolution." David W. Taylor Model Basin, Report 849, 1953.

[18] Dodbele, S.S.: "Effects of the Forebody Geometry on Subsonic Laminar Boundary Layer Stability." in Numerical Methods in Laminar and Turbulent Flows.", Pineridge Press, Vol. 5, Part 1, pp. 902-917, 1987.

[19] Vanderplaats, G. N: "CONMIN - A FORTRAN Program for Constrained Function Minimization- User's MANUAL." NASA TM X-62282, 1973.

[20] Gill, P E; and Murray, W: "Quasi-Newton Methods for Unconstrained Optimization." Journal of Institute of Mathematics Applications. 9(1972), pp. 91-108.

[21] Rechenberg, I: Cybernetic Solution Path of an Experimental Problem, Royal Aircraft Establishment, Library Translation 1122, Farnborough, 1965. 
[22] Maskew, B.: "Prediction of Subsonic Aerodynamic Characteristics- A Case for Low-Order Panel Methods." Journal of Aircraft, Vol. 19, No. 2, Feb. 1982, pp. 157-163.

[23] Harris, J.E.; and Blanchard, D.K.: "Computer Program for Solving Laminar, Transitional, or Turbulent Compressible Boundary-Layer Equations for Two-Dimensional and Axisymmetric Flow." NASA TM 83207, Feb 1982.

[24] Malik, M.R.: "COSAL-A Black-Box Compressible Stability Analysis Code for Transition Prediction in Three-Dimensional Boundary Layers." NASA CR-165925, May 1982.

[25] Gaster, M.: "Prediction of Linear Wave Packets in Laminar BoundaryLayers." AIAA Journal, Vol. 19, 1981, pp. 419-423.

[26] Kuethe, A.M.: "On the Stability of Flow in the Boundary Layer Near the Nose of a Blunt Body." U.S. Air Force Project RAND Research Memo. RM-1972, RAND Corp., August 28, 1957. (Available from DTIC as $\mathrm{AD} 150$ 687.)

[27] Morris, P.J.; and Byon, W.: "The Stability of the Axisymmetric Boundary-Layer on a Circular Cylinder." AIAA paper No. 82-1012, 1982.

[28] Floryan, J.M.; and Saric, W.S.: "Stability of Gortler Vortices in Boundary Layers." AIAA Journal 20 (4), 1982, pp. 316-324

[29] Hall, P.: "The Gortler Vortex Instability Mechanism in ThreeDimensional Boundary-Layers." Proceedings of Royal Society of London, Ser. A 399, 1985, pp.135-152.

[30] Shanebrook, J.R.; and Sumner, W.J.: "Entrainment Theory for Axisymmetric, Turbulent Incompressible Boundary Layers." Journal of Hydronautics, Vol. 4, Oct. 1970.

[31] Gërtler, M.: "Resistance Experiments on a Systematic Series of Streamlined Bodies of Revolution for Application to the Design of High-Speed Submarines." Report C-297, David Taylor Model Basin, NAVY Dep., April 1950. 


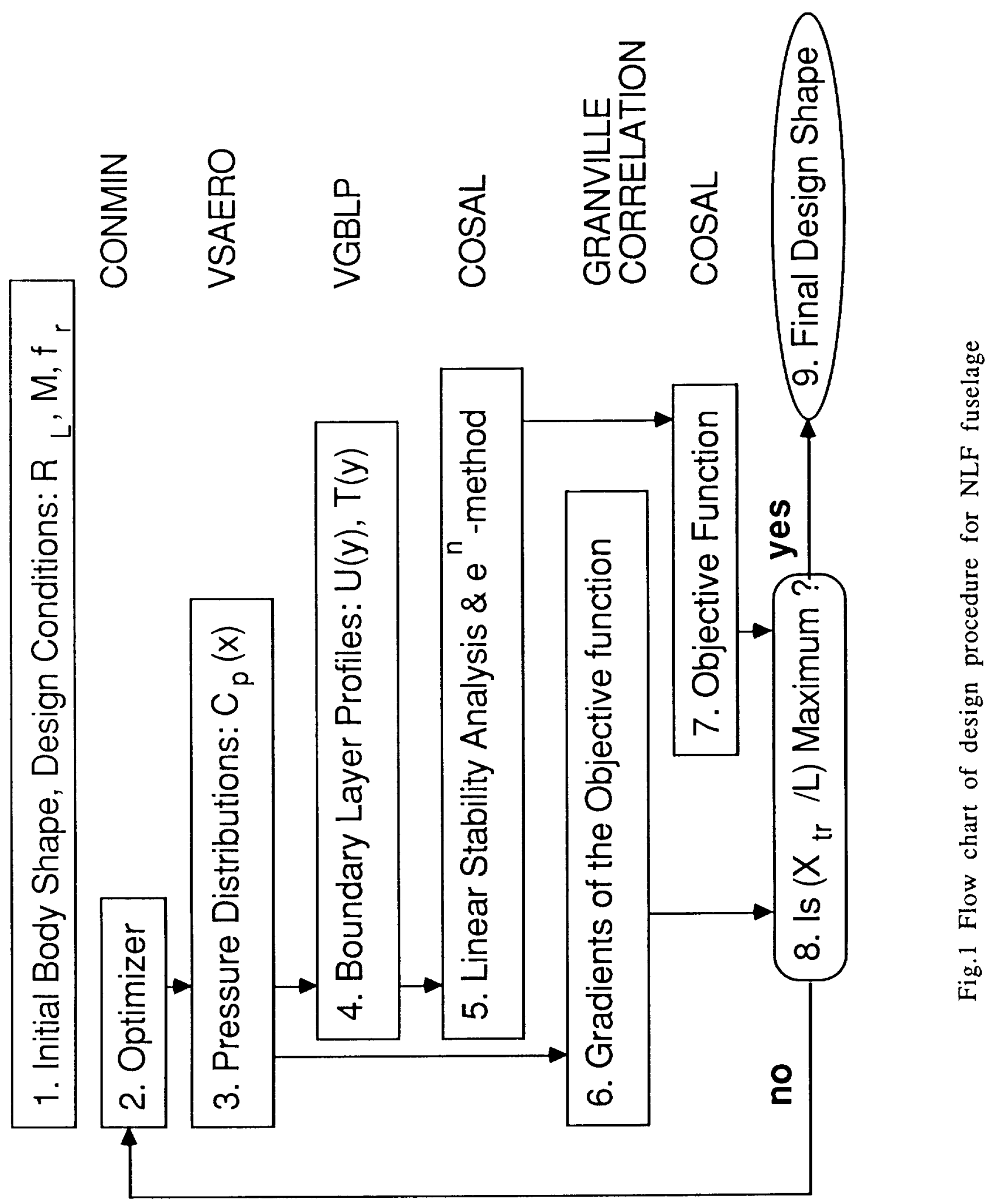




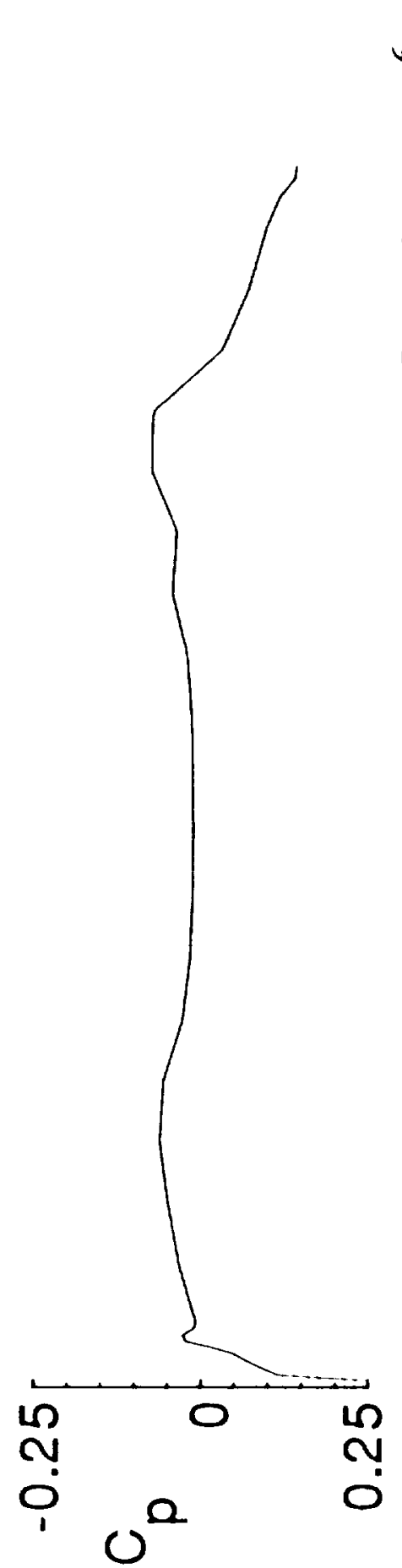

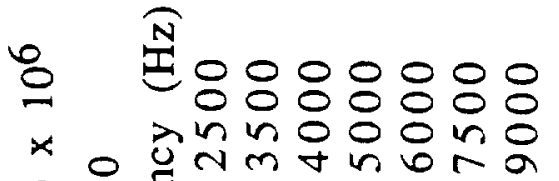

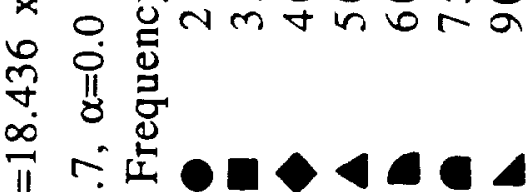

$\omega \sum^{11} \sim$

高
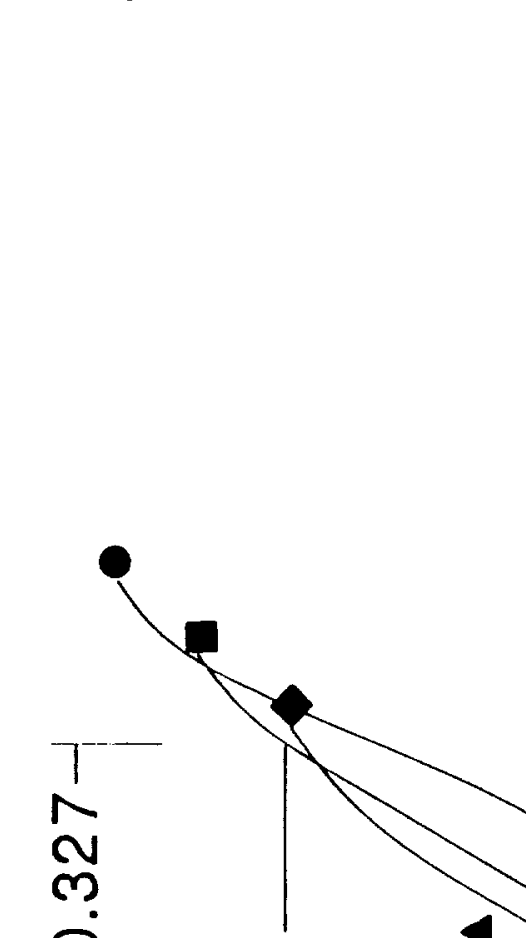

II

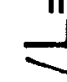

$=$

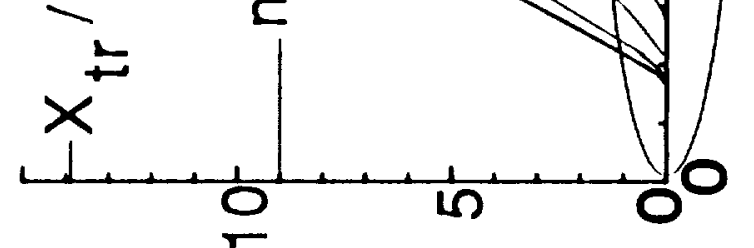

0
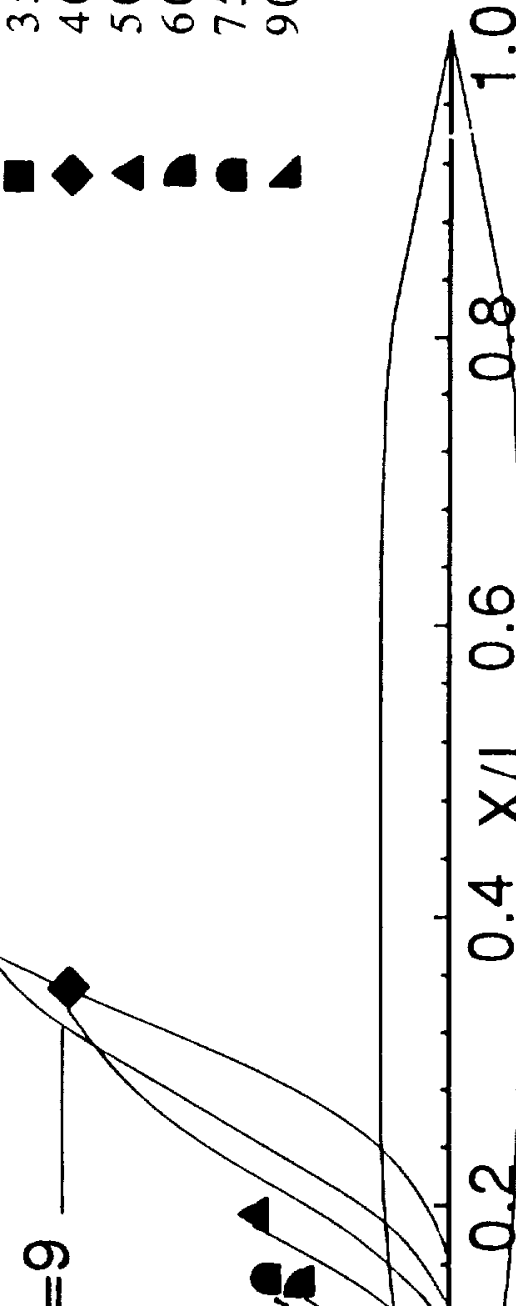

导

象

品

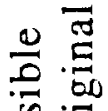

n

$d$

을

등

0

导

는

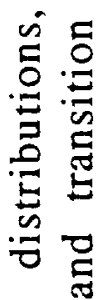

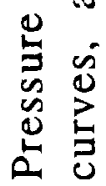

瓷

N

ס

$0^{a}$

$c$ 


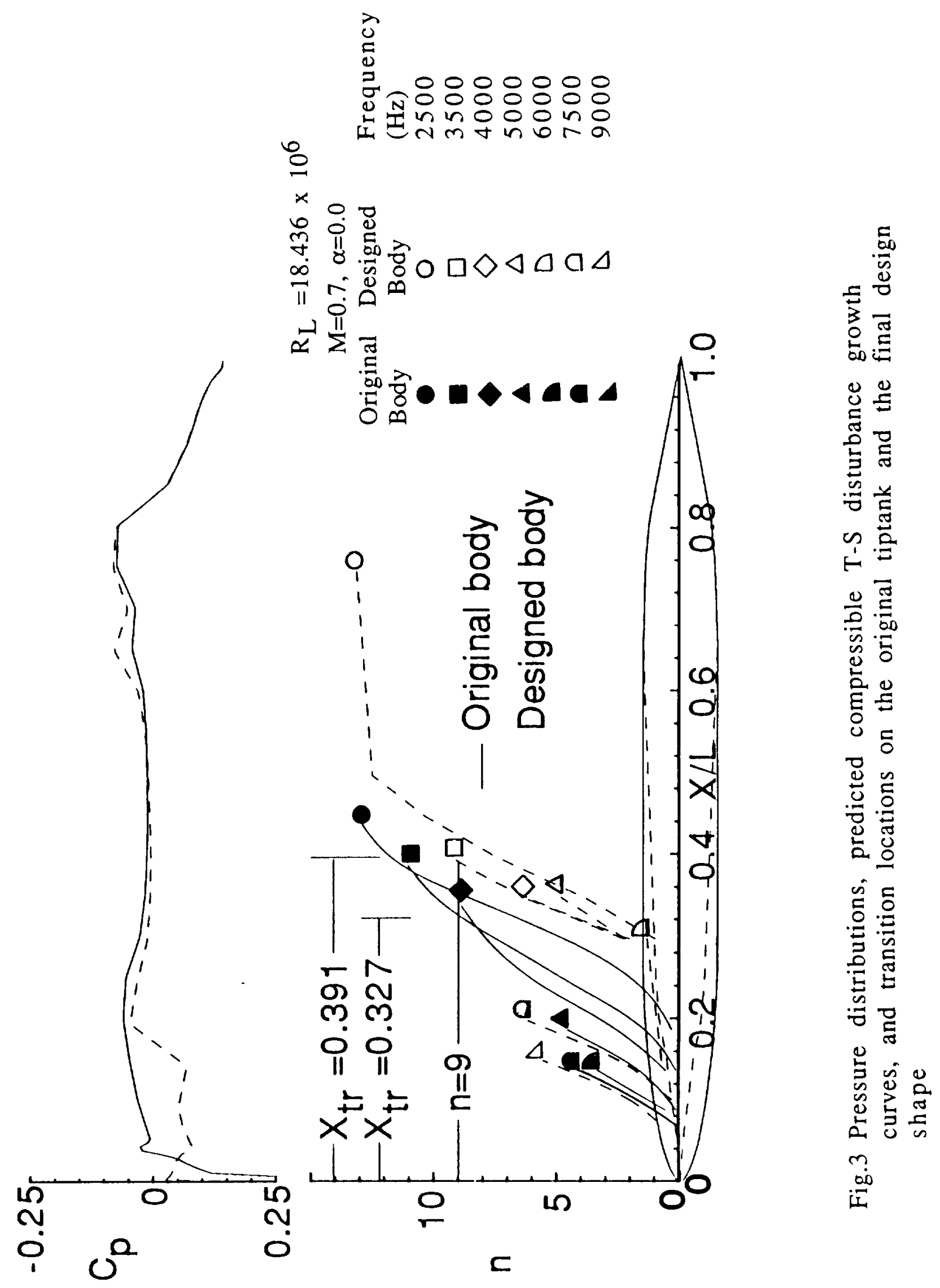




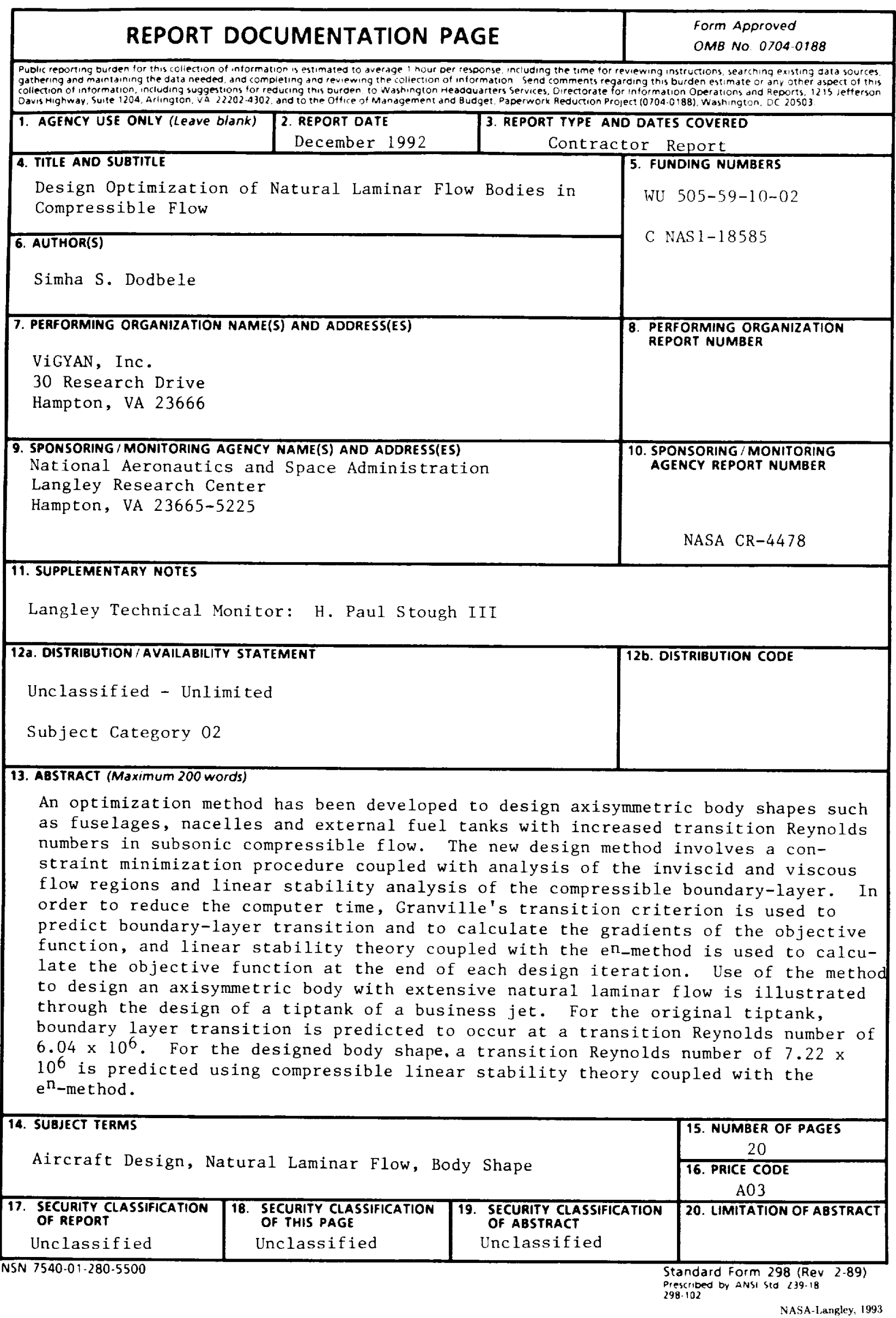


National Aeronautics and

Space Administration

Code JTT

Washington, D.C.

20546-0001

Official Business

Penalty for Private Use, $\$ 300$ 
